\title{
A half-discrete Hilbert-type inequality with a homogeneous kernel and an extension
}

\author{
Bicheng Yang ${ }^{1 *}$ and Qiang Chen ${ }^{2}$
}

* Correspondence: bcyang@gdei. edu.cn

${ }^{1}$ Department of Mathematics, Guangdong University of

Education Guangzhou, Guangdong 510303, P. R. China

Full list of author information is available at the end of the article

\section{Abstract \\ Using the way of weight functions and the technique of real analysis, a half-discrete Hilbert-type inequality with a general homogeneous kernel is obtained, and a best extension with two interval variables is given. The equivalent forms, the operator expressions, the reverses and some particular cases are considered. \\ 2000 Mathematics Subject Classification: 26D15; $47 \mathrm{~A} 07$.}

Keywords: Hilbert-type inequality, homogeneous kernel, weight function, equivalent form, reverse

\section{Introduction}

Assuming that $p>1, \frac{1}{p}+\frac{1}{q}=1, f(\geq 0) \in L^{p}\left(R_{+}\right), g(\geq 0) \in L^{q}\left(R_{+}\right)$, $\|f\|_{p}=\left\{\int_{0}^{\infty} f^{p}(x) \mathrm{d} x\right\}^{\frac{1}{p}}>0,\|g\|_{q}>0$, we have the following Hardy-Hilbert's integral inequality [1]:

$$
\int_{0}^{\infty} \int_{0}^{\infty} \frac{f(x) g(y)}{x+y} \mathrm{~d} x \mathrm{~d} y<\frac{\pi}{\sin (\pi / p)}\|f\|_{p}\|g\|_{q}
$$

where the constant factor $\frac{\pi}{\sin (\pi / p)}$ is the best possible. If $a_{m}, b_{n} \geq 0$, $b=\left\{b_{n}\right\}_{n=1}^{\infty} \in l^{q}, \quad b=\left\{b_{n}\right\}_{n=1}^{\infty} \in l^{q},\|a\|_{p}=\left\{\sum_{m=1}^{\infty} a_{m}^{p}\right\}^{\frac{1}{p}}>0,\|b\|_{q}>0$, then we still have the following discrete Hardy-Hilbert's inequality with the same best constant fac-

$$
\begin{aligned}
& \text { tor } \frac{\pi}{\sin (\pi / p)} \text { : } \\
& \qquad \sum_{m=1}^{\infty} \sum_{n=1}^{\infty} \frac{a_{m} b_{n}}{m+n}<\frac{\pi}{\sin (\pi / p)}\|a\|_{p}\|b\|_{q} .
\end{aligned}
$$

For $p=q=2$, the above two inequalities reduce to the famous Hilbert's inequalities. Inequalities (1) and (2) are important in analysis and its applications [2-4].

In 1998, by introducing an independent parameter $\lambda \in(0,1]$, Yang [5] gave an extension of (1) for $p=q=2$. Refinement and generalizing the results from [5], Yang [6] gave some best extensions of (1) and (2) as follows: If $\lambda_{1}, \lambda_{2} \in \mathbf{R}, \lambda_{1}+\lambda_{2}=\lambda, k_{\lambda}$ (x,

\section{Springer}


$y$ ) is a non-negative homogeneous function of degree $-\lambda$ satisfying for any $x, y, t>0, k_{\lambda}$ $(t x, \quad t y)=t^{-\lambda} \quad k_{\lambda} \quad(x, \quad y), \quad k\left(\lambda_{1}\right)=\int_{0}^{\infty} k_{\lambda}(t, 1) t^{\lambda_{1}-1} \mathrm{~d} t \in R_{+}, \quad \phi(x)=x^{p\left(1-\lambda_{1}\right)-1}$, $f(\geq 0) \in L_{p, \phi}\left(R_{+}\right)=\left\{f \mid\|f\|_{p, \phi}:=\left\{\int_{0}^{\infty} \phi(x)|f(x)|^{p} \mathrm{~d} x\right\}^{\frac{1}{p}}<\infty\right\}$,

$f(\geq 0) \in L_{p, \phi}\left(R_{+}\right)=\left\{f \mid\|f\|_{p, \phi}:=\left\{\int_{0}^{\infty} \phi(x)|f(x)|^{p} \mathrm{~d} x\right\}^{\frac{1}{p}}<\infty\right\}, g(\geq 0) \in L_{q, \psi},\|f\|_{p, \varphi}, \|$ $g \mid \|_{q, \psi}>0$, then we have

$$
\int_{0}^{\infty} \int_{0}^{\infty} k_{\lambda}(x, y) f(x) g(y) \mathrm{d} x \mathrm{~d} y<k\left(\lambda_{1}\right)|| f\left\|_{p, \phi}\right\| g \|_{q, \psi^{\prime}}
$$

where the constant factor $k\left(\lambda_{1}\right)$ is the best possible. Moreover, if $k_{\lambda}(x, y)$ is finite and $k_{\lambda}(x, y) x^{\lambda_{1}-1}\left(k_{\lambda}(x, y) y^{\lambda_{2}-1}\right)$ is decreasing with respect to $x>0(y>0)$, then for $a_{m}, b_{n} \geq$ $0, a=\left\{a_{m}\right\}_{m=1}^{\infty} \in l_{p, \phi}=\left\{a \mid\|a\|_{p, \phi}:=\left\{\sum_{n=1}^{\infty} \phi(n)\left|a_{n}\right|^{p}\right\}^{\frac{1}{p}}<\infty\right\}, \quad b=\left\{b_{n}\right\}_{n=1}^{\infty} \in l_{q, \psi},\|a\|$ $p, \varphi,\|b\|_{q, \Psi}>0$, we have

$$
\sum_{m=1}^{\infty} \sum_{n=1}^{\infty} k_{\lambda}(m, n) a_{m} b_{n}<k\left(\lambda_{1}\right)\|a\|_{p, \phi}\|b\|_{q, \psi}
$$

with the best constant factor $k\left(\lambda_{1}\right)$. Clearly, for $\lambda=1, k_{1}(x, y)=\frac{1}{x+y}, \lambda_{1}=\frac{1}{q}$, $\lambda_{2}=\frac{1}{p}$ (3) reduces to (1), and (4) reduces to (2). Some other results about Hilberttype inequalities are provided by [7-15].

On half-discrete Hilbert-type inequalities with the non-homogeneous kernels, Hardy et al. provided a few results in Theorem 351 of [1]. But they did not prove that the constant factors are the best possible. And, Yang [16] gave a result by introducing an interval variable and proved that the constant factor is the best possible. Recently, Yang [17] gave the following half-discrete Hilbert's inequality with the best constant factor $B\left(\lambda_{1}, \lambda_{2}\right)\left(\lambda, \lambda_{1}>0,0<\lambda_{2} \leq 1, \lambda_{1}+\lambda_{2}=\lambda\right)$ :

$$
\int_{0}^{\infty} f(x) \sum_{n=1}^{\infty} \frac{a_{n}}{(x+n)^{\lambda}} \mathrm{d} x<B\left(\lambda_{1}, \lambda_{2}\right)\|f\|_{p, \varphi}\|a\|_{q, \psi} .
$$

In this article, using the way of weight functions and the technique of real analysis, a half-discrete Hilbert-type inequality with a general homogeneous kernel and a best constant factor is given as follows:

$$
\int_{0}^{\infty} f(x) \sum_{n=1}^{\infty} k_{\lambda}(x, n) a_{n} \mathrm{~d} x<k\left(\lambda_{1}\right)|| f\left\|_{p, \varphi}\right\| a \|_{q, \psi}
$$

which is a generalization of (5). A best extension of (6) with two interval variables, some equivalent forms, the operator expressions, the reverses and some particular cases are considered. 


\section{Some lemmas}

We set the following conditions:

Condition (i) $v(y)\left(y \in\left[n_{0}-1, \infty\right)\right)$ is strictly increasing with $v\left(n_{0}-1\right) \geq 0$ and for any fixed $x \in(b, c), f(x, y)$ is decreasing for $y \in\left(n_{0}-1, \infty\right)$ and strictly decreasing in an interval of $\left(n_{0}-1, \infty\right)$.

Condition (ii) $v(y)\left(y \in\left[n_{0}-\frac{1}{2}, \infty\right)\right)$ is strictly increasing with $v\left(n_{0}-\frac{1}{2}\right) \geq 0$ and for any fixed $x \in(b, c), f(x, y)$ is decreasing and strictly convex for $y \in\left(n_{0}-\frac{1}{2}, \infty\right)$.

Condition (iii) There exists a constant $\beta \geq 0$, such that $v(y)\left(y \in\left[n_{0}-\beta, \infty\right)\right)$ is strictly increasing with $v\left(n_{0}-\beta\right) \geq 0$, and for any fixed $x \in(b, c), f(x, y)$ is piecewise smooth satisfying

$$
R(x):=\int_{n_{0}-\beta}^{n_{0}} f(x, y) \mathrm{d} y-\frac{1}{2} f\left(x, n_{0}\right)-\int_{n_{0}}^{\infty} \rho(y) f^{\prime}{ }_{\gamma}(x, y) \mathrm{d} y>0,
$$

where $\rho(y)\left(=y-[y]-\frac{1}{2}\right)$ is Bernoulli function of the first order.

Lemma 1 If $\lambda_{1}, \lambda_{2} \in \mathbf{R}, \lambda_{1}+\lambda_{2}=\lambda, k_{\lambda}(x, y)$ is a non-negative finite homogeneous function of degree $-\lambda$ in $\mathrm{R}_{+}^{2} u(x)(x \in(b, c),-\infty \leq b<c \leq \infty)$ and $v(y)\left(y \in\left[n_{0}, \infty\right), n_{0}\right.$ $\in \mathbf{N})$ are strictly increasing differential functions with $u\left(b^{+}\right)=0, v\left(n_{0}\right)>0, u\left(c^{-}\right)=v(\infty)$ $=\infty$, setting $K(x, y)=k_{\lambda}(u(x), v(y))$, then we define weight functions $\omega(n)$ and $\varpi(x)$ as follows:

$$
\begin{aligned}
& \omega(n):=[v(n)]^{\lambda_{2}} \int_{b}^{c} K(x, n)[u(x)]^{\lambda_{1}-1} u^{\prime}(x) \mathrm{d} x, n \geq n_{0}(n \in \mathbf{N}), \\
& \varpi(x):=[u(x)]^{\lambda_{1}} \sum_{n=n_{0}}^{\infty} K(x, n)[v(n)]^{\lambda_{2}-1} v^{\prime}(n), x \in(b, c) .
\end{aligned}
$$

It follows

$$
\omega(n)=k\left(\lambda_{1}\right):=\int_{0}^{\infty} k_{\lambda}(t, 1) t^{\lambda_{1}-1} \mathrm{~d} t .
$$

Moreover, setting $f(x, y):=[u(x)]^{\lambda_{1}} K(x, y)[v(y)]^{\lambda_{2}-1} v^{\prime}(y)$, if $k\left(\lambda_{1}\right) \in R_{+}$and one of the above three conditions is fulfilled, then we still have

$$
\varpi(x)<k\left(\lambda_{1}\right)(x \in(b, c)) .
$$

Proof. Setting $t=\frac{u(x)}{v(n)}$ in (7), by calculation, we have (9). 
(i) If Condition (i) is fulfilled, then we have

$$
\begin{gathered}
\varpi(x)=\sum_{n=n_{0}}^{\infty} f(x, n)<[u(x)]^{\lambda_{1}} \int_{n_{0}-1}^{\infty} K(x, y)[v(y)]^{\lambda_{2}-1} v^{\prime}(y) \mathrm{d} y \\
\stackrel{t=u(x) / v(y)}{=} \int_{0}^{\frac{u(x)}{v\left(n_{0}-1\right)}} k_{\lambda}(t, 1) t^{\lambda_{1}-1} \mathrm{~d} t \leq k\left(\lambda_{1}\right) .
\end{gathered}
$$

(ii) If Condition (ii) is fulfilled, then by Hadamard's inequality [18], we have

$$
\begin{gathered}
\varpi(x)=\sum_{n=n_{0}}^{\infty} f(x, n)<\int_{n_{0}-\frac{1}{2}}^{\infty} f(x, y) \mathrm{d} y \\
\stackrel{t=u(x) / v(\gamma)}{=} \int_{0}^{\frac{u(x)}{v\left(n_{0}-\frac{1}{2}\right)}} k_{\lambda}(t, 1) t^{\lambda_{1}-1} \mathrm{~d} t \leq k\left(\lambda_{1}\right) .
\end{gathered}
$$

(iii) If Condition (iii) is fulfilled, then by Euler-Maclaurin summation formula [6], we have

$$
\begin{aligned}
\varpi(x) & =\sum_{n=n_{0}}^{\infty} f(x, n) \\
& =\int_{n_{0}}^{\infty} f(x, y) \mathrm{d} y+\frac{1}{2} f\left(x, n_{0}\right)+\int_{n_{0}}^{\infty} \rho(y) f^{\prime}{ }_{\gamma}(x, y) \mathrm{d} y \\
& =\int_{n_{0}-\beta}^{\infty} f(x, y) \mathrm{d} y-R(x) \\
& =\frac{u(x)}{v\left(n_{0}-\beta\right)} \\
& \int_{0} k_{\lambda}(t, 1) t^{\lambda_{1}-1} \mathrm{~d} t-R(x) \\
& \left.\leq \lambda_{1}\right)-R(x)<k\left(\lambda_{1}\right) .
\end{aligned}
$$

The lemma is proved.

Lemma 2 Let the assumptions of Lemma 1 be fulfilled and additionally, $p>0(p \neq 1)$, $\frac{1}{p}+\frac{1}{q}=1, a_{n} \geq 0, n \geq n_{0}(n \in \mathbf{N}), f(x)$ is a non-negative measurable function in $(b, c)$. Then, (i) for $p>1$, we have the following inequalities:

$$
\begin{aligned}
J_{1}: & =\left\{\sum_{n=n_{0}}^{\infty} \frac{v^{\prime}(n)}{[v(n)]^{1-p \lambda_{2}}}\left[\int_{d}^{c} K(x, n) f(x) \mathrm{d} x\right]^{p}\right\}^{\frac{1}{p}} \\
& \leq\left[k\left(\lambda_{1}\right)\right]^{\frac{1}{q}}\left\{\int_{d}^{c} \varpi(x) \frac{[u(x)]^{p\left(1-\lambda_{1}\right)-1}}{\left[u^{\prime}(x)\right]^{p-1}} f^{p}(x) \mathrm{d} x\right\}^{\frac{1}{p}},
\end{aligned}
$$




$$
\begin{aligned}
L_{1}: & =\left\{\int_{b}^{c} \frac{[\varpi(x)]^{1-q} u^{\prime}(x)}{[u(x)]^{1-q \lambda_{1}}}\left[\sum_{n=n_{0}}^{\infty} K(x, n) a_{n}\right]^{q} \mathrm{~d} x\right\}^{\frac{1}{q}} \\
& \leq\left\{k\left(\lambda_{1}\right) \sum_{n=n_{0}}^{\infty} \frac{[v(n)]^{q\left(1-\lambda_{2}\right)-1}}{\left[v^{\prime}(n)\right]^{q-1}} a_{n}^{q}\right\}^{\frac{1}{q}} ;
\end{aligned}
$$

(ii) for $0<p<1$, we have the reverses of (11) and (12).

Proof (i) By Hölder's inequality with weight [18] and (9), it follows

$$
\begin{aligned}
{\left[\int_{b}^{c} K(x, n) f(x) \mathrm{d} x\right]^{p}=} & \left\{\int _ { b } ^ { c } K ( x , n ) \left[\frac{[u(x)]^{\left(1-\lambda_{1}\right) / q}}{[v(n)]^{\left(1-\lambda_{2}\right) / p}}\left[\frac{\left[v^{\prime}(n)\right]^{1 / p}}{\left.u^{\prime}(x)\right]^{1 / q}} f(x)\right]\right.\right. \\
& \times\left[\frac{[v(n)]^{\left(1-\lambda_{2}\right) / p}}{[u(x)]^{\left(1-\lambda_{1}\right) / q}}\left[\frac{\left.\left.u^{\prime}(x)\right]\right]^{1 / q}}{\left[v^{\prime}(n)\right]^{1 / p}}\right] \mathrm{d} x\right\}^{p} \\
\leq & \int_{b}^{c} K(x, n) \frac{[u(x)]^{\left[1-\lambda_{1}\right)(p-1)} v^{\prime}(n)}{[v(n)]^{1-\lambda_{2}}\left[u^{\prime}(x)\right]^{p-1} f^{p}(x) \mathrm{d} x} \\
& \times\left\{\int_{b}^{c} K(x, n) \frac{[v(n)]^{\left(1-\lambda_{2}\right)(q-1)} u^{\prime}(x)}{[u(x)]^{1-\lambda_{1}}\left[v^{\prime}(n)\right]^{q-1}} \mathrm{~d} x\right\}^{p-1} \\
= & \left\{\frac{\omega(n)[v(n)]^{q\left(1-\lambda_{2}\right)-1}}{\left[v^{\prime}(n)\right]^{q-1}}\right\}^{p-1} \int_{b}^{c} K(x, n) \frac{[u(x)]^{\left(1-\lambda_{1}\right)(p-1)} v^{\prime}(n) f^{p}(x)}{[v(n)]^{1-\lambda_{2}}\left[u^{\prime}(x)\right]^{p-1}} \mathrm{~d} x \\
= & \frac{\left[k\left(\lambda_{1}\right)\right]^{p-1}}{[v(n)]^{p p_{2}-1} v^{\prime}(n)} \int_{b}^{c} K(x, n) \frac{[u(x)]^{\left(1-\lambda_{1}\right)(p-1)} v^{\prime}(n)}{[v(n)]^{1-\lambda_{2}}\left[u^{\prime}(x)\right]^{p-1} f^{p}(x) \mathrm{d} x .}
\end{aligned}
$$

Then, by Lebesgue term-by-term integration theorem [19], we have

$$
\begin{aligned}
J_{1} & \leq\left[k\left(\lambda_{1}\right)\right]^{\frac{1}{q}}\left\{\sum_{n=n_{0}}^{\infty} \int_{b}^{c} K(x, n) \frac{[u(x)]^{\left(1-\lambda_{1}\right)(p-1)} v^{\prime}(n) f^{p}(x)}{[v(n)]^{1-\lambda_{2}}\left[u^{\prime}(x)\right]^{p-1}} \mathrm{~d} x\right\}^{\frac{1}{p}} \\
& =\left[k\left(\lambda_{1}\right)\right]^{\frac{1}{q}}\left\{\int_{b}^{c} \sum_{n=n_{0}}^{\infty} K(x, n) \frac{[u(x)]^{\left(1-\lambda_{1}\right)(p-1)} v^{\prime}(n) f^{p}(x)}{[v(n)]^{1-\lambda_{2}}\left[u^{\prime}(x)\right]^{p-1}} \mathrm{~d} x\right\}^{\frac{1}{p}} \\
& =\left[k\left(\lambda_{1}\right)\right]^{\frac{1}{q}}\left\{\int_{b}^{c} \varpi(x) \frac{[u(x)]^{p\left(1-\lambda_{1}\right)-1}}{\left[u^{\prime}(x)\right]^{p-1}} f^{p}(x) \mathrm{d} x\right\}^{\frac{1}{p}},
\end{aligned}
$$

and (11) follows.

Still by Hölder's inequality, we have

$$
\begin{aligned}
{\left[\sum_{n=n_{0}}^{\infty} K(x, n) a_{n}\right]^{q}=} & \left\{\sum_{n=n_{0}}^{\infty} K(x, n)\left[\frac{[u(x)]^{\left(1-\lambda_{1}\right) / q}}{[v(n)]^{\left[1-\lambda_{2}\right) / p}} \frac{\left[v^{\prime}(n)\right]^{1 / p}}{\left[u^{\prime}(x)\right]^{1 / q}}\right]\right. \\
& \times\left[\frac{[v(n)]^{\left(1-\lambda_{2}\right) / p}}{[u(x)]^{\left(1-\lambda_{1}\right) / q}\left[u^{\prime}(x)\right]^{1 / q}}\left[\begin{array}{l}
\left.v^{\prime}(n)\right]^{1 / p} \\
a_{n}
\end{array}\right]\right\}^{q} \\
\leq & \left\{\sum_{n=n_{0}}^{\infty} K(x, n) \frac{[u(x)]^{\left(1-\lambda_{1}\right)(p-1)}}{[v(n)]^{1-\lambda_{2}}} \frac{v^{\prime}(n)}{\left[u^{\prime}(x)\right]^{p-1}}\right\}^{q-1} \\
& \times \sum_{n=n_{0}}^{\infty} K(x, n) \frac{[v(n)]^{\left(1-\lambda_{2}\right)(q-1)}}{[u(x)]^{1-\lambda_{1}}} \frac{u^{\prime}(x)}{\left[v^{\prime}(n)\right]^{q-1}} a_{n}^{q} \\
= & \frac{[u(x)]^{1-q \lambda_{1}}}{[\varpi(x)]^{1-q} u^{\prime}(x)} \sum_{n=n_{0}}^{\infty} K(x, n) \frac{u^{\prime}(x)}{[u(x)]^{1-\lambda_{1}}} \frac{[v(n)]^{(q-1)\left(1-\lambda_{2}\right)}}{\left[v^{\prime}(n)\right]^{q-1}} a_{n}^{q} .
\end{aligned}
$$


Then, by Lebesgue term-by-term integration theorem, we have

$$
\begin{aligned}
L_{1} & \leq\left\{\int_{b}^{c} \sum_{n=n_{0}}^{\infty} K(x, n) \frac{u^{\prime}(x)}{[u(x)]^{1-\lambda_{1}}} \frac{[v(n)]^{(q-1)\left(1-\lambda_{2}\right)}}{\left[v^{\prime}(n)\right]^{q-1}} a_{n}^{q} \mathrm{~d} x\right\}^{\frac{1}{q}} \\
& =\left\{\sum_{n=n_{0}}^{\infty}\left[[v(n)]^{\lambda_{2}} \int_{b}^{c} K(x, n) \frac{u^{\prime}(x) \mathrm{d} x}{[u(x)]^{1-\lambda_{1}}}\right] \frac{[v(n)]^{q\left(1-\lambda_{2}\right)-1}}{\left[v^{\prime}(n)\right]^{q-1}} a_{n}^{q}\right\}^{\frac{1}{q}} \\
& =\left\{\sum_{n=n_{0}}^{\infty} \omega(n) \frac{[v(n)]^{q\left(1-\lambda_{2}\right)-1}}{\left[v^{\prime}(n)\right]^{q-1}} a_{n}^{q}\right\}^{\frac{1}{q}},
\end{aligned}
$$

and then in view of (9), inequality (12) follows.

(ii) By the reverse Hölder's inequality [18] and in the same way, for $q<0$, we have the reverses of (11) and (12).

\section{Main results}

We set $\Phi(x):=\frac{[u(x)]^{p\left(1-\lambda_{1}\right)-1}}{\left[u^{\prime}(x)\right]^{p-1}}(x \in(b, c)), \quad \Psi(n):=\frac{[v(n)]^{q\left(1-\lambda_{2}\right)-1}}{\left[v^{\prime}(n)\right]^{q-1}}\left(n \geq n_{0}, n \in \mathbf{N}\right)$, wherefrom $[\Phi(x)]^{1-q}=\frac{u^{\prime}(x)}{[u(x)]^{1-q \lambda_{1}}},[\Psi(n)]^{1-p}=\frac{v^{\prime}(n)}{[v(n)]^{1-p \lambda_{2}}}$.

Theorem 1 Suppose that $\lambda_{1}, \lambda_{2} \in \mathbf{R}, \lambda_{1}+\lambda_{2}=\lambda, k_{\lambda}(x, y)$ is a non-negative finite homogeneous function of degree $-\lambda$, in $\mathrm{R}_{+}^{2} u(x)(x \in(b, c),-\infty \leq b<c \leq \infty)$ and $v(y)$ $\left(y \in\left[n_{0}, \infty\right), n_{0} \in \mathbf{N}\right.$ are strictly increasing differential functions with $u\left(b^{+}\right)=0, v\left(n_{0}\right)$ $>0, u\left(c^{-}\right)=v(\infty)=\infty, \varpi(x)<k\left(\lambda_{1}\right) \in R_{+}(x \in(b, c))$. If $p>1, \frac{1}{p}+\frac{1}{q}=1, f(x), a_{n} \geq 0, f$ $L L_{p \Phi}(b, c), a=\left\{a_{n}\right\}_{n=n_{0}}^{\infty} \in l_{q, \Psi},\|f\|_{p, \Phi}>0$ and $\|a\|_{q, \Psi}>0$, then we have the following equivalent inequalities:

$$
\begin{aligned}
I: & =\sum_{n=n_{0}}^{\infty} a_{n} \int_{b}^{c} K(x, n) f(x) \mathrm{d} x=\int_{b}^{c} f(x) \sum_{n=n_{0}}^{\infty} K(x, n) a_{n} \mathrm{~d} x \\
& <k\left(\lambda_{1}\right)\|f\|_{p, \Phi}\|a\|_{q, \Psi} \\
J & :=\left\{\sum_{n=n_{0}}^{\infty}[\Psi(n)]^{1-p}\left[\int_{b}^{c} K(x, n) f(x) \mathrm{d} x\right]^{p}\right\}^{\frac{1}{p}}<k\left(\lambda_{1}\right)\|f\|_{p, \Phi}, \\
L: & =\left\{\int_{b}^{c}[\Phi(x)]^{1-q}\left[\sum_{n=n_{0}}^{\infty} K(x, n) a_{n}\right]^{q} \mathrm{~d} x\right\}^{\frac{1}{q}}<k\left(\lambda_{1}\right)\|a\|_{q, \Psi} .
\end{aligned}
$$

Moreover, if $\frac{v^{\prime}(\gamma)}{v(y)}\left(y \geq n_{0}\right)$ is decreasing and there exist constants $\delta<\lambda_{1}$ and $M>0$, such that $k_{\lambda}(t, 1) \leq \frac{M}{t^{\delta}}\left(t \in\left(0, \frac{1}{v\left(n_{0}\right)}\right]\right)$, then the constant factor $k\left(\lambda_{1}\right)$ in the above inequalities is the best possible. 
Proof By Lebesgue term-by-term integration theorem, there are two expressions for $I$ in (13). In view of (11), for $\varpi(x)<k\left(\lambda_{1}\right) \in R_{+}$, we have (14). By Hölder's inequality, we have

$$
I=\sum_{n=n_{0}}^{\infty}\left[\Psi^{\frac{-1}{q}}(n) \int_{b}^{c} K(x, n) f(x) \mathrm{d} x\right]\left[\Psi^{\frac{1}{q}}(n) a_{n}\right] \leq J\|a\|_{q, \Psi} .
$$

Then, by (14), we have (13). On the other hand, assuming that (13) is valid, setting

$$
a_{n}:=[\Psi(n)]^{1-p}\left[\int_{b}^{c} K(x, n) f(x) \mathrm{d} x\right]^{p-1}, n \geq n_{0},
$$

then $J^{p-1}=\|a\|_{q, \Psi}$. By (11), we find $J<\infty$. If $J=0$, then (14) is naturally valid; if $J$ $>0$, then by (13), we have

$$
\|a\|_{q, \Psi}^{q}=J^{p}=I<k\left(\lambda_{1}\right)\|f\|_{p, \Phi}\|a\|_{q, \Psi},\|a\|_{q, \Psi}^{q-1}=J<k\left(\lambda_{1}\right)\|f\|_{p, \Phi}
$$

and we have (14), which is equivalent to (13).

In view of (12), for $[\varpi(x)]^{1-q}>\left[k\left(\lambda_{1}\right)\right]^{1-q}$, we have (15). By Hölder's in equality, we find

$$
I=\int_{b}^{c}\left[\Phi^{\frac{1}{p}}(x) f(x)\right]\left[\Phi^{\frac{-1}{p}}(x) \sum_{n=n_{0}}^{\infty} K(x, n) a_{n}\right] \mathrm{d} x \leq\|f\|_{p, \Phi} L .
$$

Then, by (15), we have (13). On the other hand, assuming that (13) is valid, setting

$$
f(x):=[\Phi(x)]^{1-q}\left[\sum_{n=n_{0}}^{\infty} K(x, n) a_{n}\right]^{q-1}, x \in(b, c),
$$

then $L^{q}{ }_{-1}=\|f\|_{p \Phi}$. By (12), we find $L<\infty$. If $L=0$, then (15) is naturally valid; if $L$ $>0$, then by (13), we have

$$
\|f\|_{p, \Phi}^{p}=L^{q}=I<k\left(\lambda_{1}\right)\|f\|_{p, \Phi}\|a\|_{q, \Psi}\|f\|_{p, \Phi}^{p-1}=L<k\left(\lambda_{1}\right)\|a\|_{q, \Psi},
$$

and we have (15) which is equivalent to (13).

Hence, inequalities (13), (14) and (15) are equivalent.

There exists an unified constant $d \in(b, c)$, satisfying $u(d)=1$. For $0<\varepsilon<p\left(\lambda_{1}-\delta\right)$, setting $\tilde{f}(x)=0, \quad x \in(b, d) ; \tilde{f}(x)=[u(x)]^{\lambda_{1}-\frac{\varepsilon}{p}-1} u^{\prime}(x), \quad x \in(d, c)$, and $\tilde{a}_{n}=[v(n)]^{\lambda_{2}-\frac{\varepsilon}{q}-1} v^{\prime}(n), n \geq n_{0}$, if there exists a positive number $k\left(\leq k\left(\lambda_{1}\right)\right)$, such that (13) is valid as we replace $k\left(\lambda_{1}\right)$ by $k$, then in particular, we find

$$
\begin{aligned}
\tilde{I}: & =\sum_{n=n_{0}}^{\infty} \int_{b}^{c} K(x, n) \tilde{a}_{n} \tilde{f}(x) \mathrm{d} x<k \mid \tilde{f}\left\|_{p, \Phi}\right\| \tilde{a} \|_{q, \Psi} \\
& =k\left\{\int_{d}^{c} \frac{u^{\prime}(x)}{[u(x)]^{\varepsilon+1}} \mathrm{~d} x\right\}^{\frac{1}{p}}\left\{\frac{v^{\prime}\left(n_{0}\right)}{\left[v\left(n_{0}\right)\right]^{\varepsilon+1}}+\sum_{n=n_{0}+1}^{\infty} \frac{v^{\prime}(n)}{[v(n)]^{\varepsilon+1}}\right\}^{\frac{1}{q}} \\
& <k\left(\frac{1}{\varepsilon}\right)^{\frac{1}{p}}\left\{\frac{v^{\prime}\left(n_{0}\right)}{\left[v\left(n_{0}\right)\right]^{\varepsilon+1}}+\int_{n_{0}}^{\infty}[v(y)]^{-\varepsilon-1} v^{\prime}(y) \mathrm{d} y\right\} \\
& =\frac{k}{\varepsilon}\left\{\varepsilon \frac{v^{\prime}\left(n_{0}\right)}{\left[v\left(n_{0}\right)\right]^{\varepsilon+1}}+\left[v\left(n_{0}\right)\right]^{-\varepsilon}\right\}^{\frac{1}{q}}
\end{aligned}
$$




$$
\begin{aligned}
& \tilde{I}=\sum_{n=n_{0}}^{\infty}[v(n)]^{\lambda_{2}-\frac{\varepsilon}{q}-1} v^{\prime}(n) \int_{d}^{c} K(x, n)[u(x)]^{\lambda_{1}-\frac{\varepsilon}{p}-1} u^{\prime}(x) \mathrm{d} x \\
& \stackrel{t=u(x) / v(n)}{=} \sum_{n=n_{0}}^{\infty}[v(n)]^{-\varepsilon-1} v^{\prime}(n) \int_{1 / v(n)}^{\infty} k_{\lambda}(t, 1) t^{\lambda_{1}-\frac{\varepsilon}{p}-1} \mathrm{~d} t \\
& =k\left(\lambda_{1}-\frac{\varepsilon}{p}\right) \sum_{n=n_{0}}^{\infty}[v(n)]^{-\varepsilon-1} v^{\prime}(n)-A(\varepsilon) \\
& >k\left(\lambda_{1}-\frac{\varepsilon}{p}\right) \int_{n_{0}}^{\infty}[v(\gamma)]^{-\varepsilon-1} v^{\prime}(\gamma) \mathrm{d} \gamma-A(\varepsilon) \\
& =\frac{1}{\varepsilon} k\left(\lambda_{1}-\frac{\varepsilon}{p}\right)\left[v\left(n_{0}\right)\right]^{-\varepsilon}-A(\varepsilon) \text {, } \\
& A(\varepsilon):=\sum_{n=n_{0}}^{\infty}[v(n)]^{-\varepsilon-1} v^{\prime}(n) \int_{0}^{1 / v(n)} k_{\lambda}(t, 1) t^{\lambda_{1}-\frac{\varepsilon}{p}-1} \mathrm{~d} t .
\end{aligned}
$$

For $k_{\lambda}(t, 1) \leq M\left(\frac{1}{t^{\delta}}\right)\left(\delta<\lambda_{1} ; t \in\left(0,1 / v\left(n_{0}\right)\right]\right)$, we find

$$
\begin{aligned}
0<A(\varepsilon) & \leq M \sum_{n=n_{0}}^{\infty}[v(n)]^{-\varepsilon-1} v^{\prime}(n) \int_{0}^{1 / v(n)} t^{\lambda_{1}-\delta-\frac{\varepsilon}{p}-1} \mathrm{~d} t \\
& =\frac{M}{\lambda_{1}-\delta-\frac{\varepsilon}{p}} \sum_{n=n_{0}}^{\infty}[v(n)]^{-\lambda_{1}+\delta-\frac{\varepsilon}{q}-1} v^{\prime}(n) \\
& =\frac{M}{\lambda_{1}-\delta-\frac{\varepsilon}{p}}\left[\frac{v^{\prime}\left(n_{0}\right)}{\left[v\left(n_{0}\right)\right]^{\lambda_{1}-\delta+\frac{\varepsilon}{q}+1}}+\sum_{n=n_{0}+1}^{\infty} \frac{v^{\prime}(n)}{\left.v^{\prime}(n)\right]^{\lambda_{1}-\delta+\frac{\varepsilon}{q}+1}}\right] \\
& \leq \frac{v^{\prime}\left(n_{0}\right)}{\lambda_{1}-\delta-\frac{\varepsilon}{p}}\left[\frac{v^{\prime}(\gamma)}{\left.\int^{2}\left(n_{0}\right)\right]^{\lambda_{1}-\delta+\frac{\varepsilon}{q}+1}}+\int_{n_{0}}^{\infty} \frac{[v(\gamma)]^{\lambda_{1}-\delta+\frac{\varepsilon}{q}+1}}{\mathrm{~d} y}\right] \\
& =\frac{M}{\lambda_{1}-\delta-\frac{\varepsilon}{p}}\left[\frac{v^{\prime}\left(n_{0}\right)}{\left[v\left(n_{0}\right)\right]^{\lambda_{1}-\delta+\frac{\varepsilon}{q}+1}}+\frac{\left[v\left(n_{0}\right)\right]^{-\lambda_{1}+\delta-\frac{\varepsilon}{q}}}{\lambda_{1}-\delta+\frac{\varepsilon}{q}}\right]<\infty,
\end{aligned}
$$

namely $A(\varepsilon)=O(1)\left(\varepsilon \rightarrow 0^{+}\right)$. Hence, by (18) and (19), it follows

$$
k\left(\lambda_{1}-\frac{\varepsilon}{p}\right)\left[v\left(n_{0}\right)\right]^{-\varepsilon}-\varepsilon O(1)<k\left\{\varepsilon \frac{v^{\prime}\left(n_{0}\right)}{\left[v\left(n_{0}\right)\right]^{\varepsilon+1}}+\left[v\left(n_{0}\right)\right]^{-\varepsilon}\right\}^{\frac{1}{q}} .
$$

By Fatou Lemma [19], we have $k\left(\lambda_{1}\right) \leq \underline{\lim }_{\varepsilon \rightarrow 0^{+}} k\left(\lambda_{1}-\frac{\varepsilon}{p}\right)$, then by (20), it follows $k\left(\lambda_{1}\right) \leq k\left(\varepsilon \rightarrow 0^{+}\right)$. Hence, $k=k\left(\lambda_{1}\right)$ is the best value of $(12)$.

By the equivalence, the constant factor $k\left(\lambda_{1}\right)$ in (14) and (15) is the best possible, otherwise we can imply a contradiction by (16) and (17) that the constant factor in (13) is not the best possible. 
Remark 1 (i) Define a half-discrete Hilbert's operator $T: L_{p, \Phi}(b, c) \rightarrow l_{p, \Psi^{1-p}}$ as: for $f$ $\in L_{p \Phi}(b, c)$, we define $T f \in l_{p, \Psi^{1-p}}$, satisfying

$$
T f(n)=\int_{b}^{c} K(x, n) f(x) d x, \quad n \geq n_{0} .
$$

Then, by (14), it follows $\|T f\|_{p . \Psi^{1-p}} \leq k\left(\lambda_{1}\right)\|f\|_{p, \Phi}$ and then $T$ is a bounded operator with $\|T\| \leq k\left(\lambda_{1}\right)$. Since, by Theorem 1 , the constant factor in (14) is the best possible, we have $\|T\|=k\left(\lambda_{1}\right)$.

(ii) Define a half-discrete Hilbert's operator $\tilde{T}: l_{q, \Psi} \rightarrow L_{q, \Phi^{1-q}}(b, c)$ as: for $a \in l_{q^{\Psi}}$, we define $\tilde{T} a \in L_{q, \Phi^{1-q}}(b, c)$, satisfying

$$
\tilde{T} a(x)=\sum_{n=n_{0}}^{\infty} K(x, n) a_{n}, x \in(b, c) .
$$

Then, by (15), it follows $\|\tilde{T} a\|_{q, \Phi^{1-q}} \leq k\left(\lambda_{1}\right)\|a\|_{q, \Psi}$ and then $\tilde{T}$ is a bounded operator with $\|\tilde{T}\| \leq k\left(\lambda_{1}\right)$. Since, by Theorem 1, the constant factor in (15) is the best possible, we have $\|\tilde{T}\|=k\left(\lambda_{1}\right)$.

In the following theorem, for $0<p<1$, or $p<0$, we still use the formal symbols of $\|f\|_{p, \tilde{\Phi}}$ and $\|a\|_{q, \Psi}$ and so on.

Theorem 2 Suppose that $\lambda_{1}, \lambda_{2} \in \mathbf{R}, \lambda_{1}+\lambda_{2}=\lambda, k_{\lambda}(x, y)$ is a non-negative finite homogeneous function of degree $-\lambda$ in $\mathbf{R}_{+}^{2}, u(x)(x \in(b, c),-\infty \leq b<c \leq \infty)$ and $v(y)\left(y \in\left[n_{0}, \infty\right)\right.$, $\left.n_{0} \in \mathbf{N}\right)$ are strictly increasing differential functions with $u\left(b^{+}\right)=0, v\left(n_{0}\right)>0, u\left(c^{-}\right)=v(\infty)=$ $\infty, k\left(\lambda_{1}\right) \in R_{+}, \theta_{\lambda}(x) \in(0,1), k\left(\lambda_{1}\right)\left(1-\theta_{\lambda}(x)\right)<\omega(x)<k\left(\lambda_{1}\right)(x \in(b, c))$. If $0<p<1$, $\frac{1}{p}+\frac{1}{q}=1, f(x), a_{n} \geq 0, \tilde{\Phi}(x):=\left(1-\theta_{\lambda}(x)\right) \Phi(x)(x \in(b, c)), 0<\|f\|_{q, \tilde{\Phi}}<\infty$ and $0<\|$ $a \|_{q, \Psi}<\infty$. Then, we have the following equivalent inequalities:

$$
\begin{aligned}
I & :=\sum_{n=n_{0}}^{\infty} \int_{b}^{c} K(x, n) a_{n} f(x) \mathrm{d} x=\int_{b}^{c} \sum_{n=n_{0}}^{\infty} K(x, n) a_{n} f(x) \mathrm{d} x \\
& =k\left(\lambda_{1}\right)\|f\|_{p, \tilde{\Phi}}\|a\|_{q, \Psi}, \\
J & :=\left\{\sum_{n=n_{0}}^{\infty}[\Psi(n)]^{1-p}\left[\int_{b}^{c} K(x, n) f(x) \mathrm{d} x\right]^{p}\right\}^{\frac{1}{p}}>k\left(\lambda_{1}\right)\|f\|_{p, \tilde{\Phi}}, \\
\tilde{L} & :=\left\{\int_{b}^{c}[\tilde{\Phi}(x)]^{1-q}\left[\sum_{n=n_{0}}^{\infty} K(x, n) a_{n}\right]^{q} \mathrm{~d} x\right\}^{\frac{1}{q}}>k\left(\lambda_{1}\right)\|a\|_{q, \Psi} .
\end{aligned}
$$

Moreover, if $\frac{v^{\prime}(\gamma)}{v(\gamma)}\left(y \geq n_{0}\right)$ is decreasing and there exist constants $\delta, \delta_{0}>0$, such that $\theta_{\lambda}(x)=O\left(\frac{1}{[u(x)]^{s}}\right)(x \in(d, c))$ and $k\left(\lambda_{1}-\delta_{0}\right) \in \mathbf{R}_{+}$, then the constant factor $k\left(\lambda_{1}\right)$ in the above inequalities is the best possible. 
Proof. In view of (9) and the reverse of (11), for $\varpi(x)>k\left(\lambda_{1}\right)\left(1-\theta_{\lambda}(x)\right)$, we have (22). By the reverse Hölder's inequality, we have

$$
I=\sum_{n=n_{0}}^{\infty}\left[\Psi^{\frac{-1}{q}}(n) \int_{b}^{c} K(x, n) f(x) \mathrm{d} x\right]\left[\Psi^{\frac{1}{q}}(n) a_{n}\right] \geq J\|a\|_{q, \Psi} .
$$

Then, by (22), we have (21). On the other hand, assuming that (21) is valid, setting $a_{n}$ as Theorem 1, then $J^{p-1}=\|a\|_{q \Psi}$. By the reverse of (11), we find $J>0$. If $J=\infty$, then (24) is naturally valid; if $J<\infty$, then by (21), we have

$$
\|a\|_{q, \Psi}^{q}=J^{p}=I>k\left(\lambda_{1}\right)\|f\|_{p, \tilde{\Phi}}\|a\|_{q, \Psi},\|a\|_{q, \Psi}^{q-1}=J>k\left(\lambda_{1}\right)\|f\|_{p, \tilde{\Phi}^{\prime}}
$$

and we have (22) which is equivalent to (21).

In view of (9) and the reverse of (12), for $[\varpi(x)]^{1-q}>\left[k\left(\lambda_{1}\right)\left(1-\theta_{\lambda}(x)\right)\right]^{1-q}(q<0)$, we have (23). By the reverse Hölder's inequality, we have

$$
I=\int_{b}^{c}\left[\tilde{\Phi}^{\frac{1}{p}}(x) f(x)\right]\left[\tilde{\Phi}^{\frac{-1}{p}}(x) \sum_{n=n_{0}}^{\infty} K(x, n) a_{n}\right] \mathrm{d} x \geq\|f\|_{p, \tilde{\Phi}} \tilde{L} .
$$

Then, by (23), we have (21). On the other hand, assuming that (21) is valid, setting

$$
f(x):=[\tilde{\Phi}(x)]^{1-q}\left[\sum_{n=n_{0}}^{\infty} K(x, n) a_{n}\right]^{q-1}, \quad x \in(b, c),
$$

then $\tilde{L}^{q-1}=|| f \|_{p, \tilde{\Phi}}$. By the reverse of (12), we find $\tilde{L}>0$. If $\tilde{L}=\infty$, then (23) is naturally valid; if $\tilde{L}<\infty$, then by (21), we have

$$
\|f\|_{p, \tilde{\Phi}}^{p}=\tilde{L}^{q}=I>k\left(\lambda_{1}\right)|| f\left\|_{p, \tilde{\Phi}}\right\| a\left\|_{q, \Psi},\right\| f\left\|_{p, \tilde{\Phi}}^{p-1}=\tilde{L}>k\left(\lambda_{1}\right)\right\| a \|_{q, \Psi},
$$

and we have (23) which is equivalent to (21).

Hence, inequalities (21), (22) and (23) are equivalent.

For $0<\varepsilon<p \delta_{0}$, setting $\tilde{f}(x)$ and $\tilde{a}_{n}$ as Theorem 1 , if there exists a positive number $k\left(\geq k\left(\lambda_{1}\right)\right)$, such that (21) is still valid as we replace $k\left(\lambda_{1}\right)$ by $k$, then in particular, for $q$ $<0$, in view of (9) and the conditions, we have

$$
\begin{aligned}
\tilde{I}: & =\int_{b}^{c} \sum_{n=n_{0}}^{\infty} K(x, n) \tilde{a}_{n} \tilde{f}(x) \mathrm{d} x>k|| \tilde{f}\left\|_{p, \tilde{\Phi}}\right\| \tilde{a} \|_{q, \Psi} \\
& =k\left\{\int_{d}^{c}\left(1-O\left(\frac{1}{[u(x)]^{\delta}}\right)\right) \frac{u^{\prime}(x) \mathrm{d} x}{[u(x)]^{\varepsilon+1}}\right\}^{\frac{1}{p}}\left\{\sum_{n=n_{0}}^{\infty} \frac{v^{\prime}(n)}{[v(n)]^{\varepsilon+1}}\right\}^{\frac{1}{q}} \\
& =k\left\{\frac{1}{\varepsilon}-O(1)\right\}^{\frac{1}{p}}\left\{\frac{v^{\prime}\left(n_{0}\right)}{\left[v\left(n_{0}\right)\right]^{\varepsilon+1}}+\sum_{n=n_{0}+1}^{\infty} \frac{v^{\prime}(n)}{[v(n)]^{\varepsilon+1}}\right\}^{\frac{1}{q}} \\
& >k\left\{\frac{1}{\varepsilon}-O(1)\right\}^{\frac{1}{p}}\left\{\frac{v^{\prime}\left(n_{0}\right)}{\left[v\left(n_{0}\right)\right]^{\varepsilon+1}}+\int_{n_{0}}^{\infty} \frac{v^{\prime}(y)}{[v(\gamma)]^{+1}} \mathrm{~d} y\right\}^{\frac{1}{q}} \\
& =\frac{k}{\varepsilon}\{1-\varepsilon O(1)\}^{\frac{1}{p}}\left\{\frac{v^{\prime}\left(n_{0}\right)}{\left[v\left(n_{0}\right)\right]^{\varepsilon+1}}+\left[v\left(n_{0}\right)\right]^{-\varepsilon}\right\}^{\frac{1}{q}},
\end{aligned}
$$




$$
\begin{aligned}
\tilde{I} & =\sum_{n=n_{0}}^{\infty}[v(n)]^{\lambda_{2}-\frac{\varepsilon}{q}-1} v^{\prime}(n) \int_{d}^{c} K(x, n)[u(x)]^{\lambda_{1}-\frac{\varepsilon}{p}-1} u^{\prime}(x) \mathrm{d} x \\
\leq & \sum_{n=n_{0}}^{\infty}[v(n)]^{\lambda_{2}-\frac{\varepsilon}{q}-1} v^{\prime}(n) \int_{b}^{c} K(x, n)[u(x)]^{\lambda_{1}-\frac{\varepsilon}{p}-1} u^{\prime}(x) \mathrm{d} x \\
& \stackrel{t=u(x) / v(n)}{=} \sum_{n=n_{0}}^{\infty}[v(n)]^{-\varepsilon-1} v^{\prime}(n) \int_{0}^{\infty} k_{\lambda}(t, 1) t^{\left(\lambda_{1}-\frac{\varepsilon}{p}\right)-1} \mathrm{~d} t \\
\leq & k\left(\lambda_{1}-\frac{\varepsilon}{p}\right)\left[\frac{v^{\prime}\left(n_{0}\right)}{\left[v\left(n_{0}\right)\right]^{\varepsilon+1}}+\int_{n_{0}}^{\infty}[v(\gamma)]^{-\varepsilon-1} v^{\prime}(\gamma) \mathrm{d} y\right] \\
& =\frac{1}{\varepsilon} k\left(\lambda_{1}-\frac{\varepsilon}{p}\right)\left[\varepsilon \frac{v^{\prime}\left(n_{0}\right)}{\left[v\left(n_{0}\right)\right]^{\varepsilon+1}}+\left[v\left(n_{0}\right)\right]^{-\varepsilon}\right] .
\end{aligned}
$$

Since we have $k_{\lambda}(t, 1) t^{\lambda_{1}-\frac{\varepsilon}{p}-1} \leq k_{\lambda}(t, 1) t^{\lambda_{1}-\delta_{0}-1}, t \in(0,1]$ and

$$
\int_{0}^{1} k_{\lambda}(t, 1) t^{\lambda_{1}-\delta_{0}-1} \mathrm{~d} t \leq k\left(\lambda_{1}-\delta_{0}\right)<\infty
$$

then by Lebesgue control convergence theorem [19], it follows

$$
\begin{aligned}
k\left(\lambda_{1}-\frac{\varepsilon}{p}\right) & \leq \int_{1}^{\infty} k_{\lambda}(t, 1) t^{\lambda_{1}-1} \mathrm{~d} t+\int_{0}^{1} k_{\lambda}(t, 1) t^{\lambda_{1}-\frac{\varepsilon}{p}-1} \mathrm{~d} t \\
& =k\left(\lambda_{1}\right)+o(1)\left(\varepsilon \rightarrow 0^{+}\right) .
\end{aligned}
$$

By (26) and (27), we have

$$
\left(k\left(\lambda_{1}\right)+o(1)\right)\left[\varepsilon \frac{v^{\prime}\left(n_{0}\right)}{\left[v\left(n_{0}\right)\right]^{\varepsilon+1}}+\left[v\left(n_{0}\right)\right]^{-\varepsilon}\right]>k\{1-\varepsilon O(1)\}^{\frac{1}{p}}\left[\varepsilon \frac{v^{\prime}\left(n_{0}\right)}{\left[v\left(n_{0}\right)\right]^{\varepsilon+1}}+\left[v\left(n_{0}\right)\right]^{-\varepsilon}\right]^{\frac{1}{q}},
$$

and then $k\left(\lambda_{1}\right) \geq k\left(\varepsilon \rightarrow 0^{+}\right)$. Hence, $k=k\left(\lambda_{1}\right)$ is the best value of (21).

By the equivalence, the constant factor $k\left(\lambda_{1}\right)$ in (22) and (23) is the best possible, otherwise we can imply a contradiction by (24) and (25) that the constant factor in (21) is not the best possible.

In the same way, for $p<0$, we also have the following theorem.

Theorem 3 Suppose that $\lambda_{1}, \lambda_{2} \in \mathbf{R}, \lambda_{1}+\lambda_{2}=\lambda, k_{\lambda}(x, y)$ is a non-negative finite homogeneous function of degree $-\lambda$, in $\mathbf{R}_{+}^{2}, u(x)(x \in(b, c),-\infty \leq b<c \leq \infty)$ and $v(y)(y$ $\left.\in\left[n_{0}, \infty\right), n_{0} \in \mathbf{N}\right)$ are strictly increasing differential functions with $u\left(b^{+}\right)=0, v\left(n_{0}\right)>0$, $u\left(c^{-}\right)=v(\infty)=\infty, \varpi(x)<k\left(\lambda_{1}\right) \in R_{+}(x \in(b, c))$. If $p<0, \frac{1}{p}+\frac{1}{q}=1, f(x), a_{n} \geq 0,0<\| f$ \|\|$_{p \Phi}<\infty$ and $0<\|a\|_{q, \Psi}<\infty$. Then, we have the following equivalent inequalities:

$$
\begin{aligned}
I & :=\sum_{n=n_{0}}^{\infty} \int_{b}^{c} K(x, n) a_{n} f(x) \mathrm{d} x=\int_{b}^{c} \sum_{n=n_{0}}^{\infty} K(x, n) a_{n} f(x) \mathrm{d} x \\
& >k\left(\lambda_{1}\right)\|f\|_{p, \Phi}\|a\|_{q, \Psi},
\end{aligned}
$$




$$
\begin{aligned}
& J:=\left\{\sum_{n=n_{0}}^{\infty}[\Psi(n)]^{1-p}\left[\int_{b}^{c} K(x, n) f(x) \mathrm{d} x\right]^{p}\right\}^{\frac{1}{p}}>k\left(\lambda_{1}\right)\|f\|_{p, \Phi,} \\
& \tilde{L}:=\left\{\int_{b}^{c}[\Phi(x)]^{1-q}\left[\sum_{n=n_{0}}^{\infty} K(x, n) a_{n}\right]^{q} \mathrm{~d} x\right\}^{\frac{1}{q}}>k\left(\lambda_{1}\right)\|a\|_{q, \Psi} .
\end{aligned}
$$

Moreover, if $\frac{v^{\prime}(\gamma)}{v(y)}\left(y \geq n_{0}\right)$ is decreasing and there exists constant $\delta_{0}>0$, such that $k\left(\lambda_{1}\right.$ $\left.+\delta_{0}\right) \in \mathbf{R}_{+}$, then the constant factor $k\left(\lambda_{1}\right)$ in the above inequalities is the best possible.

Remark 2 (i) For $n_{0}=1, b=0, c=\infty, u(x)=v(x)=x$, if

$$
\varpi(x)=x^{\lambda_{1}} \sum_{n=1}^{\infty} k_{\lambda}(x, n) n^{\lambda_{2}-1}<k\left(\lambda_{1}\right) \in \mathbf{R}_{+}(x \in(0, \infty)),
$$

then (13) reduces to (6). In particular, for $k_{\lambda}(x, n)=\frac{1}{(x+n)^{\lambda}}\left(\lambda=\lambda_{1}+\lambda_{2}, \lambda_{1}>0,0<\lambda_{2} \leq 1\right),(6)$ reduces to (5).

(ii) For $n_{0}=1, b=0, c=\infty, u(x)=v(x)=x^{\alpha}(\alpha>0)$, $k_{\lambda}(x, y)=\frac{1}{(\max \{x, y\})^{\lambda}}\left(\lambda, \lambda_{1}>0,0<\alpha \lambda_{2} \leq 1\right)$, since

$$
f(x, y)=\frac{\alpha x^{\alpha \lambda_{1}} \gamma^{\alpha \lambda_{2}-1}}{\left(\max \left\{x^{\alpha}, \gamma^{\alpha}\right\}\right)^{\lambda}}=\left\{\begin{array}{l}
\alpha x^{-\alpha \lambda_{2}} \gamma^{\alpha \lambda_{2}-1}, y \leq x \\
\alpha x^{\alpha \lambda_{1}} y^{-\alpha \lambda_{1}-1}, y>x
\end{array}\right.
$$

is decreasing for $y \in(0, \infty)$ and strictly decreasing in an interval of $(0, \infty)$, then by Condition (i), it follows

$$
\begin{gathered}
\varpi(x)<\alpha x^{\alpha \lambda_{1}} \int_{0}^{\infty} \frac{1}{\left(\max \left\{x^{\alpha}, y^{\alpha}\right\}\right)^{\lambda}} \gamma^{\alpha\left(\lambda_{2}-1\right)} y^{\alpha-1} \mathrm{~d} y \\
\stackrel{t=(\gamma / x)^{\alpha}}{\int_{0}^{\infty}} \frac{t^{\lambda_{2}-1}}{(\max \{1, t\})^{\lambda}} \mathrm{d} t=\frac{\lambda}{\lambda_{1} \lambda_{2}}=k\left(\lambda_{1}\right) .
\end{gathered}
$$

Since for $\delta=\frac{\lambda_{1}}{2}<\lambda_{1}, k_{\lambda}(t, 1)=1 \leq \frac{1}{t^{\delta}}(t \in(0,1])$, then by (13), we have the following inequality with the best constant factor $\frac{\lambda}{\alpha \lambda_{1} \lambda_{2}}$ :

$$
\begin{aligned}
& \sum_{n=1}^{\infty} a_{n} \int_{0}^{\infty} \frac{1}{\left(\max \left\{x^{\alpha}, n^{\alpha}\right\}\right)^{\lambda}} f(x) \mathrm{d} x \\
< & \frac{\lambda}{\alpha \lambda_{1} \lambda_{2}}\left\{\int_{0}^{\infty} x^{p\left(1-\alpha \lambda_{1}\right)-1} f^{p}(x) \mathrm{d} x\right\}^{\frac{1}{p}}\left\{\sum_{n=1}^{\infty} n^{q\left(1-\alpha \lambda_{2}\right)-1} a_{n}^{q}\right\}^{\frac{1}{q}} .
\end{aligned}
$$

(iii) For $n_{0}=1, \quad b=\beta, c=\infty, \quad u(x)=v(x)=x-\beta\left(0 \leq \beta \leq \frac{1}{2}\right)$, $k_{\lambda}(x, y)=\frac{\ln (x / y)}{x^{\lambda}-\gamma^{\lambda}}\left(\lambda, \lambda_{1}>0,0<\lambda_{2} \leq 1\right)$, since for any fixed $x \in(\beta, \infty)$, 


$$
f(x, y)=(x-\beta)^{\lambda_{1}} \frac{\ln [(x-\beta) /(y-\beta)]}{(x-\beta)^{\lambda}-(y-\beta)^{\lambda}}(y-\beta)^{\lambda_{2}-1}
$$

is decreasing and strictly convex for $y \in\left(\frac{1}{2}, \infty\right)$, then by Condition (ii), it follows

$$
\begin{gathered}
\varpi(x)<\quad(x-\beta)^{\lambda_{1}} \int_{\frac{1}{2}}^{\infty} \frac{\ln [(x-\beta) /(y-\beta)]}{(x-\beta)^{\lambda}-(y-\beta)^{\lambda}}(y-\beta)^{\lambda_{2}-1} \mathrm{~d} y \\
\underset{t=[(y-\beta) /(x-\beta)]^{\lambda}}{=} \frac{1}{\lambda^{2}} \int_{\sum^{\frac{1}{2}-\beta}}^{\infty} \frac{\ln t}{t-1} t^{\left(\lambda_{2} / \lambda\right)-1} \mathrm{~d} t \\
\leq \quad \frac{1}{\lambda^{2}-\beta} \int_{0}^{\infty} \frac{\ln t}{t-1} t^{\left(\lambda_{2} / \lambda\right)-1} \mathrm{~d} t=\left[\frac{\pi}{\lambda \sin \left(\frac{\pi \lambda_{1}}{\lambda}\right)}\right]^{2} .
\end{gathered}
$$

Since for $\delta=\frac{\lambda_{1}}{2}<\lambda_{1}, k_{\lambda}(t, 1)=\frac{l n t}{t^{\lambda}-1} \leq \frac{M}{t^{\delta}}(t \in(0,1])$, then by (13), we have the following inequality with the best constant factor $\left[\frac{\pi}{\lambda \sin \left(\frac{\pi \lambda_{1}}{\lambda}\right)}\right]^{2}$ :

$$
\begin{aligned}
& \sum_{n=1}^{\infty} a_{n} \int_{\beta}^{\infty} \frac{\ln [(x-\beta) /(n-\beta)]}{(x-\beta)^{\lambda}-(n-\beta)^{\lambda}} f(x) \mathrm{d} x<\left[\frac{\pi}{\lambda \sin \left(\frac{\pi \lambda_{1}}{\lambda}\right)}\right]^{2} \\
& \times\left\{\int_{\beta}^{\infty}(x-\beta)^{p\left(1-\lambda_{1}\right)-1} f^{p}(x) \mathrm{d} x\right\}^{\frac{1}{p}}\left\{\sum_{n=1}^{\infty}(n-\beta)^{q\left(1-\lambda_{2}\right)-1} a_{n}^{q}\right\}^{\frac{1}{q}} .
\end{aligned}
$$

(iv) For $n_{0}=1, b=1-\beta=\gamma, c=\infty, u(x)=v(x)=(x-\gamma)$, $k_{\lambda}(x, y)=\frac{1}{x^{\lambda}+y^{\lambda}}(0<\lambda \leq 4), k_{\lambda}(x, y)=\frac{1}{x^{\lambda}+y^{\lambda}}(0<\lambda \leq 4), \lambda_{1}=\lambda_{2}=\frac{\lambda}{2}$, we have

$$
\begin{aligned}
k\left(\frac{\lambda}{2}\right) & =\int_{0}^{\infty} k_{\lambda}(t, 1) t^{\frac{\lambda}{2}-1} \mathrm{~d} t=\frac{2}{\lambda} \int_{0}^{\infty} \frac{1}{t^{\lambda}+1} \mathrm{~d} t^{\frac{\lambda}{2}} \\
& =\left.\frac{2}{\lambda} \arctan t^{\frac{\lambda}{2}}\right|_{0} ^{\infty}=\frac{\pi}{\lambda} \in \mathbf{R}_{+}
\end{aligned}
$$

and

$$
f(x, y)=\frac{(x-\gamma)^{\frac{\lambda}{2}}(y-\gamma)^{\frac{\lambda}{2}-1}}{(x-\gamma)^{\lambda}+(y-\gamma)^{\lambda}}(x, y \in(\gamma, \infty)) .
$$

Hence, $v(y)(y \in[\gamma, \infty))$ is strictly increasing with $v(1-\beta)=v(\gamma)=0$, and for any fixed $x \in(\gamma, \infty), f(x, y)$ is smooth with

$$
f_{\gamma}^{\prime}(x, y)=-\left(1+\frac{\lambda}{2}\right) \frac{(x-\gamma)^{\frac{\lambda}{2}}(y-\gamma)^{\frac{\lambda}{2}-2}}{(x-\gamma)^{\lambda}+(y-\gamma)^{\lambda}}+\frac{\lambda(x-\gamma)^{\frac{3 \lambda}{2}}(y-\gamma)^{\frac{\lambda}{2}-2}}{\left[(x-\gamma)^{\lambda}+\left(y-\gamma^{\lambda}\right]^{2}\right.} .
$$


We set

$$
R(x):=\int_{\gamma}^{1} f(x, y) \mathrm{d} y-\frac{1}{2} f(x, 1)-\int_{1}^{\infty} \rho(y) f_{\gamma}^{\prime}(x, y) \mathrm{d} y .
$$

For $x \in(\gamma, \infty), 0<\lambda \leq 4$, by (33) and the following improved Euler-Maclaurin summation formula [6]:

$$
\frac{-1}{8} g(1)<\int_{1}^{\infty} \rho(y) g(y) \mathrm{d} y<0\left((-1)^{i} g^{(i)}(\gamma)>0, g^{(i)}(\infty)=0, i=0,1\right),
$$

we have

$$
\begin{aligned}
& R(x)=\int_{\gamma}^{1} \frac{(x-\gamma)^{\frac{\lambda}{2}}(y-\gamma)^{\frac{\lambda}{2}-1}}{(x-\gamma)^{\lambda}+(\gamma-\gamma)^{\lambda}} \mathrm{d} y-\frac{1}{2} \frac{(x-\gamma)^{\frac{\lambda}{2}}(1-\gamma)^{\frac{\lambda}{2}-1}}{(x-\gamma)^{\lambda}+(1-\gamma)^{\lambda}} \\
&+\left(1+\frac{\lambda}{2}\right) \int_{1}^{\infty} \rho(\gamma) \frac{(x-\gamma)^{\frac{\lambda}{2}}(y-\gamma)^{\frac{\lambda}{2}-2}}{(x-\gamma)^{\lambda}+(\gamma-\gamma)^{\lambda}} \mathrm{d} \gamma \\
& \quad-\int_{1}^{\infty} \rho(\gamma) \frac{\lambda(x-\gamma)^{\frac{3 \lambda}{2}}(y-\gamma)^{\frac{\lambda}{2}-2}}{\left[(x-\gamma)^{\lambda}+\left(\gamma-\gamma^{\lambda}\right]^{2}\right.} \mathrm{d} \gamma \\
&> \frac{2}{\lambda} \arctan \left(\frac{1-\gamma}{x-\gamma}\right)^{\frac{\lambda}{2}}-\frac{(1-\gamma)^{\frac{\lambda}{2}-1}(x-\gamma)^{\frac{\lambda}{2}}}{2\left[(1-\gamma)^{\lambda}+\left(x-\gamma^{\lambda}\right]\right.} \\
&-\frac{1}{8}\left(1+\frac{\lambda}{2}\right) \frac{(1-\gamma)^{\frac{\lambda}{2}-2}(x-\gamma)^{\frac{\lambda}{2}}}{(1-\gamma)^{\lambda}+(x-\gamma)^{\lambda}}+0 \\
&= h(x):=\frac{2}{\lambda} \arctan \left(\frac{1-\gamma}{x-\gamma}\right)^{\frac{\lambda}{2}} \\
&-\left[\frac{1-\gamma}{2}+\frac{1}{8}\left(1+\frac{\lambda}{2}\right)\right] \frac{(1-\gamma)^{\frac{\lambda}{2}-2}(x-\gamma)^{\frac{\lambda}{2}}}{(1-\gamma)^{\lambda}+(x-\gamma)^{\lambda}} .
\end{aligned}
$$

Since for $\gamma \leq 1-\frac{1}{8}[\lambda+\sqrt{\lambda(3 \lambda+4)}]$, i.e. $1-\gamma \geq \frac{1}{8}[\lambda+\sqrt{\lambda(3 \lambda+4)}](0<\lambda \leq 4)$,

$$
\begin{aligned}
h^{\prime}(x)=\frac{(1-\gamma)^{\frac{\lambda}{2}}(x-\gamma)^{-\frac{\lambda}{2}-1}}{(1-\gamma)^{\lambda}+(x-\gamma)^{\lambda}}-\left[\frac{1-\gamma}{2}+\frac{1}{8}\left(1+\frac{\lambda}{2}\right)\right](1-\gamma)^{\frac{\lambda}{2}-2} \\
\quad \times\left\{\frac{\lambda(x-\gamma)^{\frac{\lambda}{2}-1}}{2\left[(1-\gamma)^{\lambda}+(x-\gamma)^{\lambda}\right]}-\frac{\lambda(x-\gamma)^{\frac{3 \lambda}{2}-1}}{\left[(1-\gamma)^{\lambda}+(x-\gamma)^{\lambda}\right]^{2}}\right\} \\
=-\left[(1-\gamma)^{2}-\frac{\lambda}{4}(1-\gamma)-\frac{\lambda}{16}\left(1+\frac{\lambda}{2}\right)\right] \frac{(1-\gamma)^{\frac{\lambda}{2}-2}(x-\gamma)^{\frac{\lambda}{2}-1}}{(1-\gamma)^{\lambda}+(x-\gamma)^{\lambda}} \\
-\left[\frac{1-\gamma}{2}+\frac{1}{8}\left(1+\frac{\lambda}{2}\right)\right] \frac{\lambda(1-\gamma)^{\frac{3 \lambda}{2}-2}(x-\gamma)^{\frac{\lambda}{2}-1}}{\left[(1-\gamma)^{\lambda}+(x-\gamma)^{\lambda}\right]^{2}}<0,
\end{aligned}
$$

then $h(x)$ is strictly decreasing and $R(x)>h(x)>h(\infty)=0$. 
Then, by Condition (iii), it follows $\varpi(x)<k\left(\frac{\lambda}{2}\right)=\frac{\pi}{\lambda}(x \in(\gamma, \infty))$. For $\delta=0<\frac{\lambda}{2}$, it follows

$$
k_{\lambda}(t, 1)=\frac{1}{t^{\lambda}+1} \leq 1=\frac{1}{t^{\delta}}, \quad t \in\left(0, \frac{1}{1-\gamma}\right),
$$

and by (13), we have the following inequality with the best constant factor $\frac{\pi}{\lambda}$ :

$$
\begin{aligned}
& \sum_{n=1}^{\infty} a_{n} \int_{\gamma}^{\infty} \frac{1}{(x-\gamma)^{\lambda}+(n-\gamma)^{\lambda}} f(x) \mathrm{d} x \\
& <\frac{\pi}{\lambda}\left\{\int_{\gamma}^{\infty}(x-\gamma)^{p\left(1-\frac{\lambda}{2}\right)-1} f^{p}(x) \mathrm{d} x\right\}^{\frac{1}{p}}\left\{\sum_{n=1}^{\infty}(n-\gamma)^{q\left(1-\frac{\lambda}{2}\right)-1} a_{n}^{q}\right\}^{\frac{1}{q}},
\end{aligned}
$$

where $\gamma \leq 1-\frac{1}{8}[\lambda+\sqrt{\lambda(3 \lambda+4)}](0<\lambda \leq 4)$.

\section{Acknowledgements}

This study was supported by the Guangdong Science and Technology Plan Item (No. 2010B010600018) and Guangdong Modern Information Service Industry Develop Particularly item 2011 (No. 13090).

\section{Author details}

${ }^{1}$ Department of Mathematics, Guangdong University of Education Guangzhou, Guangdong 510303, P. R. China

${ }^{2}$ Department of Computer Science, Guangdong University of Education Guangzhou, Guangdong 510303, P. R. China

\section{Authors' contributions}

BY wrote and reformed the article. QC conceived of the study, and participated in its design and coordination. All authors read and approved the final manuscript.

\section{Competing interests}

The authors declare that they have no competing interests.

Received: 28 August 2011 Accepted: 30 November 2011 Published: 30 November 2011

\section{References}

1. Hardy, GH, Littlewood, JE, Pólya, G: Inequalities. Cambridge University Press, Cambridge, UK (1934)

2. Mitrinović, DS, Pečarić, JE, Fink, AM: Inequalities Involving Functions and their Integrals and Derivatives. Kluwer Academic Publishers, Boston, MA (1991)

3. Yang, B: Hilbert-Type Integral Inequalities. Bentham Science Publishers Ltd. Arabia Unites a Tribal Chief Country (2009)

4. Yang, B: Discrete Hilbert-Type Inequalities. Bentham Science Publishers Ltd. Arabia Unites a Tribal Chief Country (2011)

5. Yang, B: On Hilbert's integral inequality. J Math Anal Appl. 271, 778-785 (1998)

6. Yang, B: The Norm of Operator and Hilbert-Type Inequalities. Science Press, Beijing, China (2009)

7. Yang, B, Brnetić, I, Krnić, M, Pečarić, J: Generalization of Hilbert and Hardy-Hilbert integral inequalities. Math Inequal Appl. 8(2), 259-272 (2005)

8. Krnić, M, Pečarić, J: Hilbert's inequalities and their reverses. Publ Math Debrecen. 67(3-4), 315-331 (2005)

9. Jin, J, Debnath, L: On a Hilbert-type linear series operator and its applications. J Math Anal Appl. 371, 691-704 (2010). doi:10.1016/j.jmaa.2010.06.002

10. Azar, L: On some extensions of Hardy-Hilbert's inequality and applications. J Inequal Appl. 2009(Article ID 546829), 12 (2006)

11. Yang, B, Rassias, T: On the way of weight coefficient and research for Hilbert-type inequalities. Math Inequal Appl. 6(4), 625-658 (2003)

12. Arpad, B, Choonghong, O: Best constant for certain multilinear integral operator. J Inequal Appl. 2006(Article ID 28582), $12(2006)$

13. Kuang, J, Debnath, L: On Hilbert's type inequalities on the weighted Orlicz spaces. Pacific J Appl Math. 1(1), 95-103 (2007)

14. Zhong, W: The Hilbert-type integral inequality with a homogeneous kernel of Lambda-degree. J Inequal Appl. 2008(Article ID 917392), 13 (2008)

15. Li, Y, He, B: On inequalities of Hilbert's type. Bull Aust Math Soc. 76(1), 1-13 (2007). doi:10.1017/S0004972700039423

16. Yang, B: A mixed Hilbert-type inequality with a best constant factor. Int J Pure Appl Math. 20(3), 319-328 (2005)

17. Yang, B: A half-discrete Hilbert's inequality. J Guangdong Univ Edu. 31(3), 1-7 (2011) 
Cite this article as: Yang and Chen: A half-discrete Hilbert-type inequality with a homogeneous kernel and an extension. Journal of Inequalities and Applications 2011 2011:124.

Submit your manuscript to a SpringerOpen ${ }^{\odot}$ journal and benefit from:

- Convenient online submission

$\checkmark$ Rigorous peer review

- Immediate publication on acceptance

- Open access: articles freely available online

- High visibility within the field

- Retaining the copyright to your article

Submit your next manuscript at $\boldsymbol{\nabla}$ springeropen.com 